\title{
Histologic changes of the fetal membranes after fetoscopic laser surgery for twin-twin transfusion syndrome
}

\author{
Ramesha Papanna ${ }^{1,2}$, Lovepreet K. Mann², Kenneth J. Moise Jr. ${ }^{2}$, Themis Kyriakides ${ }^{3}$, Anthony Johnson², Elisa Garcia², \\ Catalin S. Buhimschi ${ }^{4}$ and Irina A. Buhimschi ${ }^{4-6}$
}

\begin{abstract}
BACKGROUND: Preterm premature rupture of membranes remains a major complication after fetoscopic laser surgery (FLS) for twin-twin transfusion syndrome (TTTS). We studied the histologic changes of fetal membranes post-FLS and investigated a possible impact of amniotic fluid (AF) dilution.

METHODS: Fetal membranes of 31 pregnancies that underwent FLS for TTTS were investigated histologically at delivery at different sites: trocar site of recipient sac and at distance, donor sac, and inter-twin membrane.
\end{abstract}

RESULTS: The trocar insertion site on the recipient sac showed no signs of histologic hallmarks of healing. Wide-spread alteration in collagen organization and higher apoptotic index in the amnion of the recipient sac which were absent in donor's and reference membranes. To explain the mechanisms, we analyzed the AF composition of recipient sacs from TTTS pregnancies vs. GA-matched healthy singleton controls and found glucose, protein and lactate dehydrogenase activity were all significantly lower in TTTS sacs consistent with over-dilution of recipient's AF ( 2-fold). In-vitro exposure of healthy amniochorion to analogous dilutional stress conditions recapitulated the histologic changes and induced apoptosis and autophagy.

CONCLUSION: Alteration in structural integrity of the recipient's amniochorion, possibly in response to dilution stress, along with ineffective repair mechanisms may explain the increased incidence of preterm birth post-FLS.

D espite the success of fetoscopic laser surgery (FLS) for the treatment of severe twin-twin transfusion (TTTS), preterm delivery with or without preterm premature rupture of membranes (PPROM) remains a major complication (1-4). The average gestational age at delivery after procedure ranges between 29 to $33 \mathrm{wk}(1,5)$. Aside from the cost of caring for the premature infants, preterm delivery is directly associated with long-term neurological deficits in TTTS $(4,6)$, undermining the complete benefits of such intervention. PPROM which affects $20-32 \%$ of cases is the most important risk factor leading to preterm delivery $(2,7-10)$.
Considered a minimally invasive procedure, FLS necessitates the insertion of a trocar into the amniotic cavity of the recipient twin to allow for insertion of the fetoscope (through a cannula which stays in place throughout the procedure) (11). A laser is used to seal the abnormal blood vessel anastomoses between the monochorionic twin placentas. Lastly, the surgeon performs an amnioreduction to relieve the recipient's polyhydramnios by draining the excess amniotic fluid (AF) through the cannula before removing it. During the procedure to allow proper visualization due to the inadvertent loss of amniotic fluid through cannula port or due to cloudy AF, it is customary for the surgeon to remove a volume of $\mathrm{AF}$ and replace it via amnioinfusion with a solution of Lactated Ringer's (LR).

Fetal membranes are the outermost layer of fetal compartment in apposition to maternal decidua. The amniotic layer of the fetal membranes is made up of a lining of cuboidal epithelial cells attached to a thick basement membrane over a collagenous matrix with a fibroblast layer. The outermost layer is the chorion, comprised of a layer of extravillous trophoblasts (cEVTs) embedded in a loose stroma with minimal amounts of collagen $(12,13)$. A shared histological feature of both structures is their lack of vascular supply implying that in any event of injury the process of healing cannot follow traditional mechanisms of repair (13). Although not specifically tested, it then becomes more natural for fetal membranes to follow the repair sequence of other avascular human tissues such as cornea and tympanic membrane where the key event is the migration of the epithelium adjacent to the site of injury across the defect (14-16).

In the absence of vascular supply, amnion epithelial cells in the fetal membranes derive nutrients from the $\operatorname{AF}(17,18)$. One study has shown that when placed in culture, amnion epithelial cells are capable of endocytosis and autophagy (selfeating) to adapt to the abrupt changes in the environment (19). For many cell types, the process of autophagy has been well-studied in relation to cellular adaptation to stress and as means to delay apoptosis in low nutrient conditions $(20,21)$. However, it is unknown whether cells in the fetal membranes 
turn to autophagy or apoptosis in response to changes in AF concentration occurring in vivo such as in instances of polyhydramnios (e.g., the recipient sac of pregnancies with TTTS) or postamnioinfusion during FLS procedures. The objectives of this study were: (i) to explore systematically the array of histological changes of the fetal membranes occurring post-FLS for TTTS both at the trocar insertion site and at distance; (ii) to explore whether over-dilution of AF on the recipient side which is a common phenomenon in TTTS pregnancies could be mechanistically linked to histologic changes in the amniochorion and to the high incidence of PPROM post-FLS.

\section{RESULTS}

\section{Demographic and Clinical Characteristics of the Patients}

Demographics, surgical, and outcome characteristics of TTTS cases that contributed tissues $(n=31)$ and/or AF for biochemical analysis $(n=24)$ are presented in Table 1 .

\section{Gross Morphologic Features and Clinical Correlates of the Fetal Membranes}

Figure 1a is a schematic representation of the gestational sac marking the sites where fetal membranes were collected for histologic examination relative to the trocar insertion site. Although the size of the defect varied, a patent hole was clearly identified in all 31 cases where the fetal membranes were surveyed. Figure $\mathbf{1 b}$ displays the gross morphology of a representative fetal membrane defect found at delivery. Other than the thickened rounded edges, there was little macroscopic evidence of tissue remodeling. In the majority of these cases, the defect's area measured was in excess or the same as the cross-sectional area of the cannula. Interestingly, the area of the defect correlated inversely with the procedure-to-delivery interval. In multivariable regression analysis, the procedureto-delivery interval was associated with the combination of the GA at procedure $(r=-0.489 ; P<0.001)$ and the area of the surgically induced defect in the fetal membranes as measured at the time of delivery $(r=-0.572 ; P<0.001)$. The earlier the GA at procedure and larger the defect, the shorter the interval to delivery (multivariate $R^{2}=0.554 ; P<0.001$ ). Excluded from the model based on $P>0.1$ were the size of the cannula and method of entry (direct vs. Seldinger's).

\section{Histologic Analysis of the Fetal Membranes at the Trocar Entry Site}

When examined by H\&E and Masson's trichrome, the site of trocar entry showed no evidence of neovascularization at the edge of defect and no evidence of extension of the amnion epithelium over the excisional wound (Figure 1c,d, green arrow). Myofibroblasts were absent in the amnion's compact, fibroblast, and spongiosum layers. This implies that a key feature of wound healing, contraction, is absent in the fetal membranes. Additionally, no inflammatory infiltrate was observed. The architecture of the fetal membranes was remarkably distorted at the defect site. There was abundant collagen deposition with loss of the normal wavy pattern and increased number of collagen whorls. The cellular component
Table 1. Characteristics of TTTS cases that contributed fetal membranes for histologic analyses and/or AF for biochemical analysis

\begin{tabular}{|c|c|c|}
\hline Characteristics & $\begin{array}{l}\text { Histologic analysis, } \\
\qquad n=31\end{array}$ & $\begin{array}{c}\text { AF analysis cases, } \\
n=24\end{array}$ \\
\hline Maternal age, years ${ }^{\mathrm{a}}$ & $28 \pm 5$ & $31 \pm 6$ \\
\hline Gravidity $^{\mathrm{b}}$ & $2(1-7)$ & $2(1-5)$ \\
\hline Parity $^{\mathrm{b}}$ & $0(0-4)$ & $0(0-3)$ \\
\hline History of preterm birth ${ }^{c}$ & $2(6.5 \%)$ & $0(0 \%)$ \\
\hline Gestational age at FLS, weeks ${ }^{\mathrm{a}}$ & $21 \pm 3$ & $20 \pm 3$ \\
\hline Growth discordance, $\mathrm{g}^{\mathrm{a}}$ & $26 \pm 11$ & $23 \pm 12$ \\
\hline Anterior placentac & $13(42 \%)$ & $9(36 \%)$ \\
\hline \multicolumn{3}{|l|}{ TTTS severity, stage ${ }^{c}$} \\
\hline Stage I & $7(23 \%)$ & $4(16 \%)$ \\
\hline Stage II & $10(32 \%)$ & $8(33 \%)$ \\
\hline Stage III & $13(42 \%)$ & $12(50 \%)$ \\
\hline Stage IV & $4(13 \%)$ & $0(0 \%)$ \\
\hline \multicolumn{3}{|l|}{ Maximum vertical pocket, $\mathrm{cm}^{\mathrm{a}}$} \\
\hline Onset of procedure & $11 \pm 2$ & $12 \pm 3$ \\
\hline End of procedure & $7 \pm 1$ & $8 \pm 2$ \\
\hline \multicolumn{3}{|l|}{ Trocar outer diameter ${ }^{c}$} \\
\hline $10($ French scale $)=3.33(\mathrm{~mm})$ & $13(42 \%)$ & $13(54 \%)$ \\
\hline $12($ French scale $)=4(\mathrm{~mm})$ & $18(58 \%)$ & $18(11 \%)$ \\
\hline Amnioreduction volume, $\mathrm{ml}^{\mathrm{b}}$ & $2000(340-5,000)$ & $2000(300-5,000)$ \\
\hline Amnioinfusion volume, $\mathrm{ml}^{\mathrm{b}}$ & $800(100-4,600)$ & $960(100-5,200)$ \\
\hline $\begin{array}{l}\text { Chorio-amnion separation } \\
\text { post-FLSc }\end{array}$ & $4(13 \%)$ & $3(13 \%)$ \\
\hline PPROM at $<34 w^{c}$ & $9(29 \%)$ & $10(42 \%)$ \\
\hline $\begin{array}{l}\text { Procedure-to-delivery interval, } \\
\text { days }^{\mathrm{b}}\end{array}$ & $79(0-113)$ & $70(0-120)$ \\
\hline $\begin{array}{l}\text { Gestational age at delivery, } \\
\text { weeks }^{\mathrm{a}}\end{array}$ & $31 \pm 4$ & $31 \pm 3$ \\
\hline \multicolumn{3}{|l|}{$\begin{array}{l}\text { Gestational age at delivery } \\
\text { periodsc }\end{array}$} \\
\hline$<25^{0 / 7} w k$ & $3(10 \%)$ & $2(8 \%)$ \\
\hline $25^{0 / 7}-29^{6 / 7} w k$ & $7(22 \%)$ & $6(25 \%)$ \\
\hline $30^{0 / 7}-33^{6 / 7} w k$ & $18(58 \%)$ & $12(50 \%)$ \\
\hline $34^{0 / 7}-36^{6 / 7} \mathrm{wk}$ & $3(10 \%)$ & $3(13 \%)$ \\
\hline$\geq 37^{0 / 7} \mathrm{wk}$ (term) & $0(0 \%)$ & $1(4 \%)$ \\
\hline \multicolumn{3}{|l|}{ Live births ${ }^{c}$} \\
\hline 0 & $1(3 \%)$ & $0(0 \%)$ \\
\hline 1 & $5(16 \%)$ & $4(17 \%)$ \\
\hline 2 & $25(81 \%)$ & $20(83 \%)$ \\
\hline \multicolumn{3}{|l|}{ Birth weight, $g^{a}$} \\
\hline Donor twin & $1,435 \pm 488$ & $1,511 \pm 630$ \\
\hline Recipient twin & $1,620 \pm 511$ & $1,808 \pm 421$ \\
\hline
\end{tabular}

AF, amniotic fluid; FLS, fetal laser surgery; PPROM, preterm premature rupture of membranes; TTTS, twin-twin transfusion syndrome.

Data presented as ${ }^{\mathrm{a}}$ mean $\pm \mathrm{SD},{ }^{\mathrm{b}}$ median (range), or ${ }^{\mathrm{c}} n(\%)$. 
a

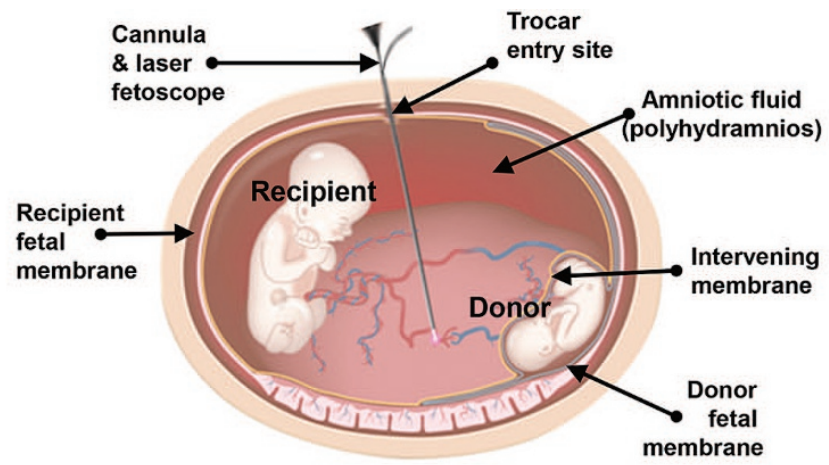

b

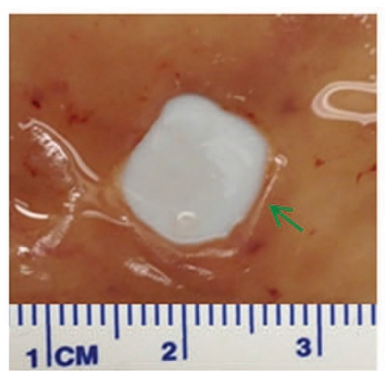

c

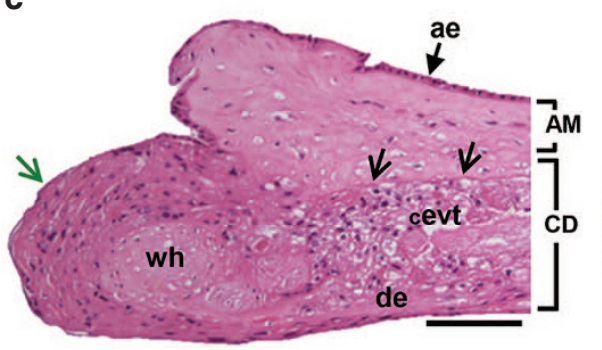

d

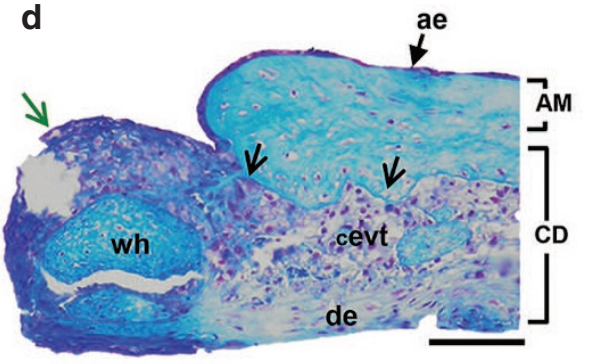

e

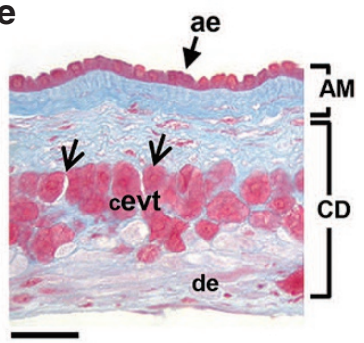

Figure 1. Twin-twin transfusion syndrome (TTTS) schematics and the non-healing nature of fetal membranes at the trocar site. (a) Diagram of amniotic sacs in pregnancies complicated by TTTS due to presence of placental anastomoses which are the target of fetoscopic laser photocoagulation (FLS); (b) Fetal membrane explant showing the site of defect of a $10 \mathrm{Fr}$ ( $3.3 \mathrm{~mm}$ outer diameter) trocar entry and the diameter of the defect measured $10 \mathrm{~mm}$. The edges (green arrow) appear thicker than the rest of the fetal membranes; (c)-H\&E and (d)-Masson Trichrome staining of the edges at the trocar insertion site; (e)-Trichrome staining of reference amniochorion from a preterm singleton gestation. $A M$, amnion layer of the membranes; $C D$, choriodecidua layer. Ae, amniotic epithelial cells; de, decidual cells; cevt, chorionic extravillous trophoblast cells; wh, whorl-like structures seen with increased frequency in recipient's membranes. Black arrows: basement membrane separating the amnion from the chorio-decidua, green arrows: edge of the surgically-induced defect. Scale bars: c, d: $500 \mu \mathrm{m}$ or e: $50 \mu \mathrm{m}$.

was also abnormal. Amnion epithelial cells were flattened or absent and chorionic extravillous trophoblasts (cEVTs) showed degenerated morphology with pyknotic nuclei and vacuolated cytoplasm, in stark contrast to the scarlet-red cytoplasm of cEVTs from reference amniochorion at similar GA (Figure 1e). To further understand remodeling process occurring postsurgery at the site of the trocar, we stained tissue sections with cytokeratin (epithelial marker) and noted the site of the defect heavily populated by cEVTs (Figure 2a) and not by fibroblasts (Figure $2 b$ ). The expansion of cEVTs at the site of injury occurred in the absence of significant cell proliferation ( $<1-2$ per high-power field) as indicated by the Ki-67 antigen (marker for dividing cells, Figure 2c) implying that the cEVTs actively migrated from the chorion to cover the transected edge of the fetal membranes. Appreciated better on higher magnification micrographs, an additional observation was the loss of cytokeratin positivity of the amnion cells (Figure 2d) along with gain of vimentin staining, suggestive of epithelial-mesenchymal transition (EMT) (Figure 2e) Lastly, an unexpected observation was that the derangement in cellular morphology and collagen architecture was not limited to the trocar injured area but extended to the entire length of the biopsy suggestive that perhaps the damage to the recipient's fetal membranes in TTTS cases, may involve mechanisms beyond the nonhealing nature of the membranes at the puncture site. Additionally, there were no histological

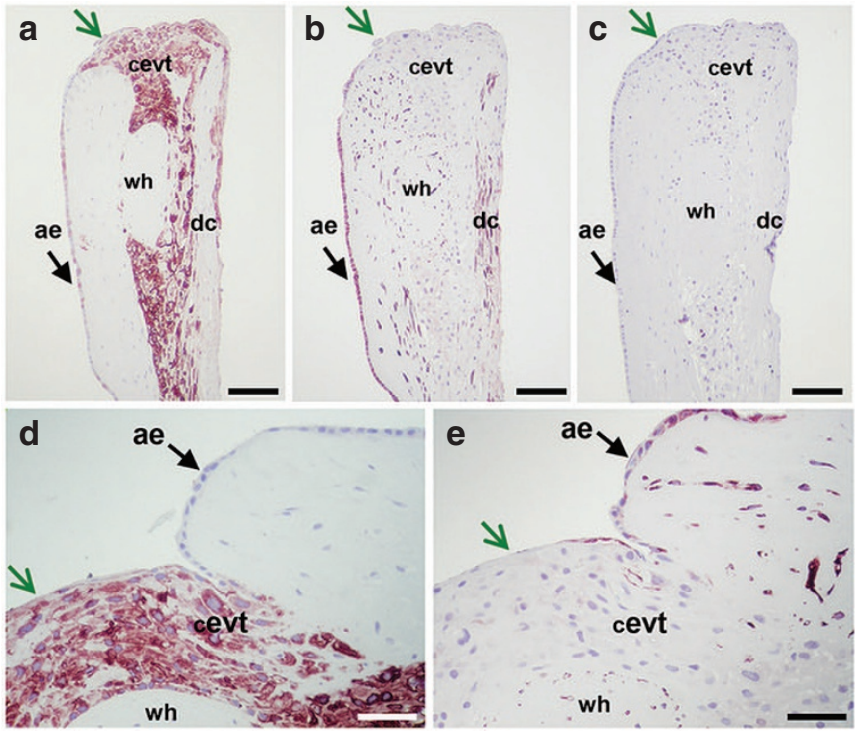

Figure 2. Characterization of cells at the trocar site using immunohistochemistry. (a and d (high magnification)) Cytokeratin staining of trocar site membrane showing the leading edge (green arrow) of the defect is formed by extravillous trophoblast (cevt); $\mathbf{b}$ and $\mathbf{e}$ (high magnification)Vimentin staining of trocar site membrane showing the leading edge (green arrow); c, Ki67 staining at the trocar site showing no cellular proliferation. ae, amniotic epithelial cells; cevt, chorionic extravillous trophoblast; dc, chorio-decidual layer; wh, whorls in the chorion. Scale bars: a-c: $100 \mu \mathrm{m}$ or d,e: $50 \mu \mathrm{m}$. 

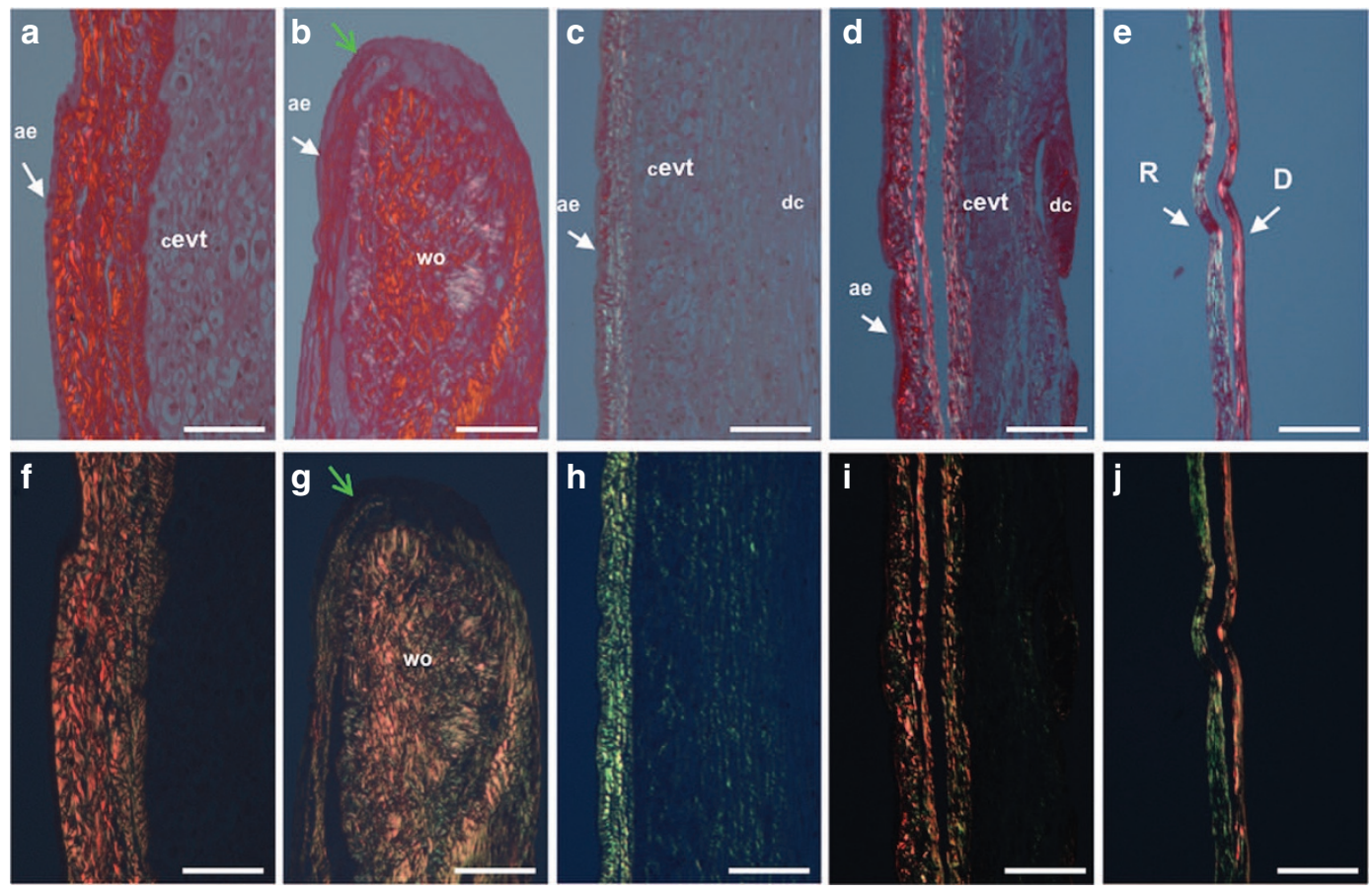

Figure 3. Representative histological sections of different sites of fetal membranes after picro-sirus red staining visualized under polarized microscopy. (a-e): white light microscopy and $\mathbf{f}-\mathbf{j}$ : cross-polarized microscopy; $\mathbf{a}, \mathbf{f}$ : fetal membranes from healthy reference singleton gestation; $\mathbf{b}, \mathbf{g}$ : Trocar site in the recipient membrane (green arrow)- edge of the defect; $\mathbf{c}, \mathbf{h}$ : Remote recipient membrane; $\mathbf{d}, \mathbf{i}$ : donor membrane and $\mathbf{e}, \mathbf{j}$ : Intervening membrane showing recipient side (R) and the donor side (D). ae, amniotic epithelial cells; cevt, chorionic extravillous trophoblast; dc, chorio-decidual layer. Scale bar d,e: $100 \mu \mathrm{m}$

evidences of chorioamnionitis such as granulocyte infiltration, in any of the fetal membranes.

\section{Collagen Orientation in the Fetal Membranes of Pregnancies Complicated by TTTS}

In Figure 3, we display representative white light (Figure 3ae) and polarized light (Figure $\mathbf{3} \mathbf{f}-\mathbf{j}$ ) images of collagen fibers after staining with Sirius Red. Control tissues displayed long wavy red-orange birefringent fibers of mature thick collagen (Figure 3a). This aspect was absent in the recipient amniotic membranes, both at the trocar (Figure $3 \mathrm{~b}, \mathrm{~g}$ ) and remote areas (Figure $3 \mathbf{c}, \mathbf{h}$ ). At trocar site, the polarized light image was dominated by fibers in disordered orientation while the remote site was characterized by thinning of the fibers indicated by predominance of green rather than red birefringence. This observation was less visible at the donor site (Figure 3d,i) which retained the red-orange collagen in parallel orientation. Remarkably, the recipient side of the intervening membrane (Figure 3e,j) displayed more abundant green birefringence (immature, thin collagen) compared to the donor side.

\section{Apoptosis in the Fetal Membranes of Pregnancies Complicated by TTTS}

When compared to reference fetal membranes (CRL, Figure 4a), TUNEL staining demonstrated increased cell death especially at the margins of the trocar entry site (green arrow, Figure $4 \mathrm{~b}$ ). Cellular apoptosis was extended to the recipient's membrane samples also remote from the trocar site (Figure 4c). Apoptotic cells were scattered throughout the layers of the amniochorion. This observation was in contrast to fetal membranes of the donor fetus where apoptotic cells were only rarely encountered (Figure $4 \mathrm{~d}$ ). The difference in apoptosis between the recipient vs. donor sac was best noted in amnion cells of the intervening membrane (Figure 4e). The results of the quantitative analysis for amnion epithelial cells, amnion mesenchymal cells and cell in the chorio-decidua are presented in Figure 4f,h,i, respectively. As shown, the highest levels of cellular apoptosis was observed at the margins of the trocar entry site and along the fetal membranes of the recipient sac (Figure 4f, $P<0.001$ vs. both CRL and donor $\mathrm{sac})$. Although the differences among sites followed a similar pattern, it appeared that amnion epithelial cells were more affected by apoptosis compared to amnion mesenchymal cells $(P=0.031)$ but not compared to choriodecidual cells $(P=0.324)$. There was no significant correlation between the apoptotic index and GA in amnion epithelial cells at delivery either in singleton CRLs $(r=-0.17 ; P=0.56)$ or in TTTS recipient remote membrane ( $r=0.03 ; P=0.89)$, suggesting no effect of GA on the cell viability. The findings were similar in amnion mesenchymal and choriodecidual layer.

AF Levels of Total Proteins, Glucose, and LDH in the Recipient Sac In Table 2, we present the results of the quantitative analysis of AF protein, glucose, and $\mathrm{LDH}$. The recipient sac of pregnancies with TTTS had significantly lower levels of total proteins, glucose and LDH, suggesting a dilutional effect on these analytes compared to that of singleton normal gestations. These data suggest that fetal membranes of the recipient are exposed even 

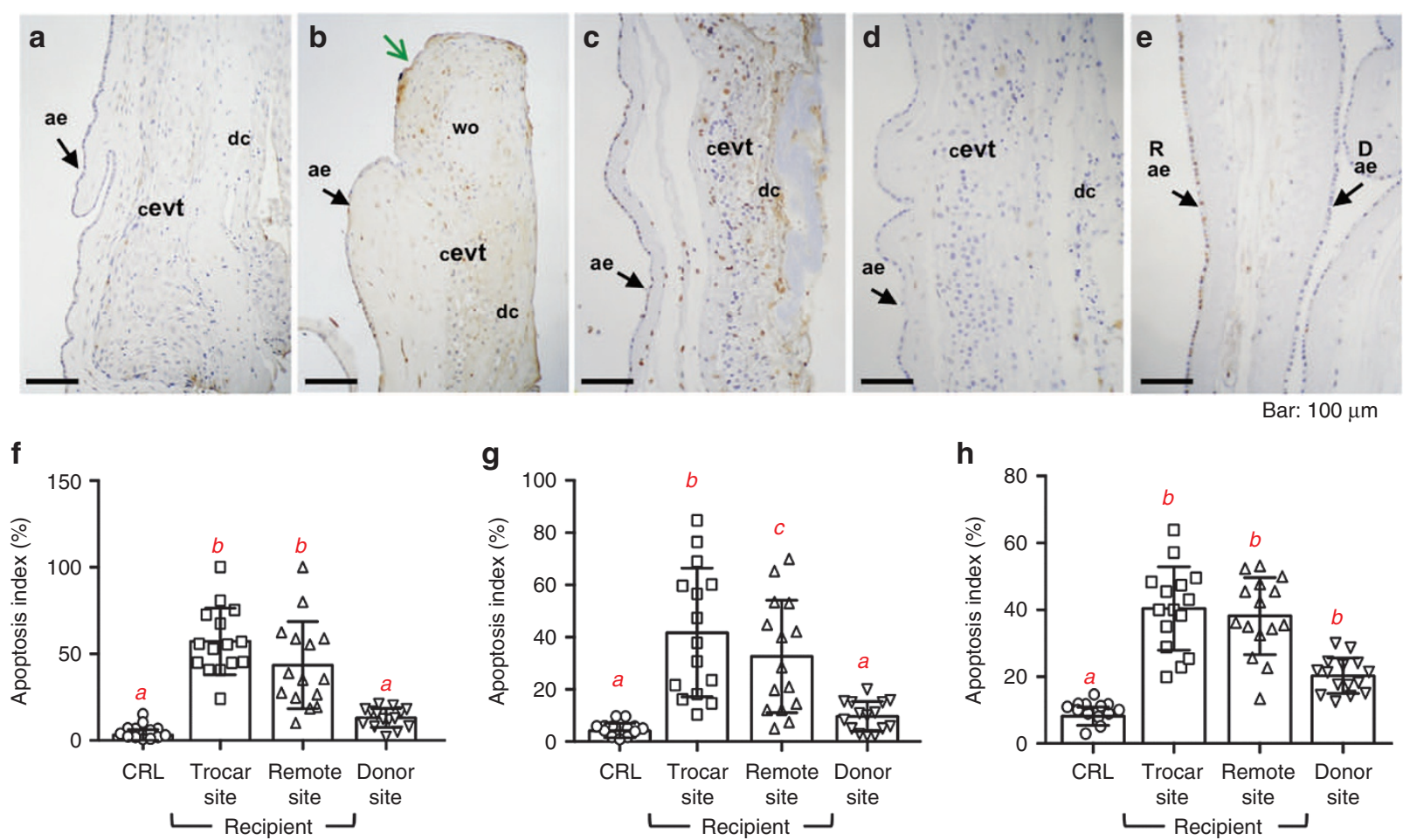

Figure 4. Representative histological sections of different sites of fetal membranes after TUNEL staining. (a) Fetal membranes from reference singleton gestation; (b) recipient trocar site; (c) recipient remote site; (d) donor fetal membrane; (e) Intervening membrane showing the recipient side (R) and the donor side (D); (f-h) graphical representation of apoptotic index for amniotic epithelial cells, amniotic mesenchymal cells, and chorio-decidual cells, respectively. The $P$ values for post-hoc comparison using Holm-Sidak method between the groups are represented with values for each comparison. Data presented as mean \pm SD and analyzed by one-way ANOVA. Groups sharing at least one common red letter are not statistically different at $P>0.05$. Scale bar a-e: $100 \mu \mathrm{m}$.

Table 2. Amniotic fluid constituents in the recipient sac before FLS compared to a group of healthy controls at similar gestational age

\begin{tabular}{lccr}
\hline Characteristics & $\begin{array}{c}\text { TTTS, } \\
n=24\end{array}$ & $\begin{array}{c}\text { Healthy } \\
\text { controls, } n=18\end{array}$ & $P$ value \\
\hline Gestational age at FLS, weeks ${ }^{\mathrm{a}}$ & $20.3 \pm 2.5$ & $19 \pm 2.3$ & 0.120 \\
Protein, $\mathrm{g} / \mathrm{l}^{\mathrm{a}}$ & $2.5 \pm 0.7$ & $4.9 \pm 1.1$ & $<0.001$ \\
Glucose, $\mathrm{mg} / \mathrm{dl}^{\mathrm{a}}$ & $31.0 \pm 9.0$ & $42.9 \pm 7.2$ & $<0.001$ \\
$\mathrm{LDH}, \mathrm{Ul} / \mathrm{dl}^{\mathrm{a}}$ & $27.5 \pm 23.1$ & $89.1 \pm 45.5$ & $<0.001$ \\
\hline
\end{tabular}

FLS, fetal laser surgery; TTTS, twin-twin transfusion syndrome.

${ }^{a}$ Data presented as mean \pm SD and compared using Student's $t$-test.

before the procedure to a different amniotic fluid composition which postprocedure will be changes even more drastically due to LR amnioinfusion.

\section{Fetal Membranes Autophagy and Apoptosis Following Tissue Incubation With AF, AF:LR, and LR Solution}

The basal autophagy activity at $0 \mathrm{~h}$ was $<1-2 \%$ of amniotic epithelial cells (Figure 5a) and was similar in explants cultured in amniotic fluid only at $6 \mathrm{~h}$ (Figure $5 \mathrm{~b}$ ). As shown, only $1-2 \%$ of amnion cells display positive immunostaining for the LC3-II antibody. In contrast, amnion cells of the fetal membranes incubated with AF diluted 1:1 with LR displayed a higher autophagy activity at $6 \mathrm{~h}$ with characteristic puncta observed around the autophagosome vacuole (green arrow, Figure 5c) and at $24 \mathrm{~h}$ compared to native AF. The explants that were cultured in LR alone showed a higher rate of autophagy compared to other two groups at both 6 (Figure 5d) and $24 \mathrm{~h}$ interval (not shown). Additionally, the explants cultured in LR alone exhibited cellular blebs on the cell membrane suggesting cell death (22). In Figure 5e, we show the graphical representation of the autophagic index with varying AF dilution and duration of incubation.

TUNEL staining of the same explants showed baseline apoptotic index of $<1-2 \%$ (Figure 5f). The apoptotic cells which appeared brown (green arrow) were seen in 5\% of amniotic epithelial cells at $6 \mathrm{~h}$ in native AF culture (Figure $5 \mathrm{~g}$ ) and were present in greater than $50 \%$ cells of AF diluted 1:1 with LR (Figure 5h). The apoptotic cells which appeared brown (green arrow) increased to $50 \%$ at $6 \mathrm{~h}$ culture in LR only solution (Figure $5 \mathrm{i}$ ). At $24 \mathrm{~h}$ of culture in LR only, the amniotic epithelial cells were completely apoptotic and in some locations the cells were detached from the basement membrane, similar to the findings in some of the recipient trocar site. In Figure 5j, it shows the graphical representation of apoptotic index with duration and dilution of different fluids.

\section{DISCUSSION}

The findings of our study may be summarized fivefold: (i) Following an invasive procedure such as FLS for TTTS, human fetal membranes do not heal. This assertion was based on the histological absence of granulation tissue, neovascularization, myofibroblast, or epithelial proliferation at the 


\section{Articles | Papannaetal.}
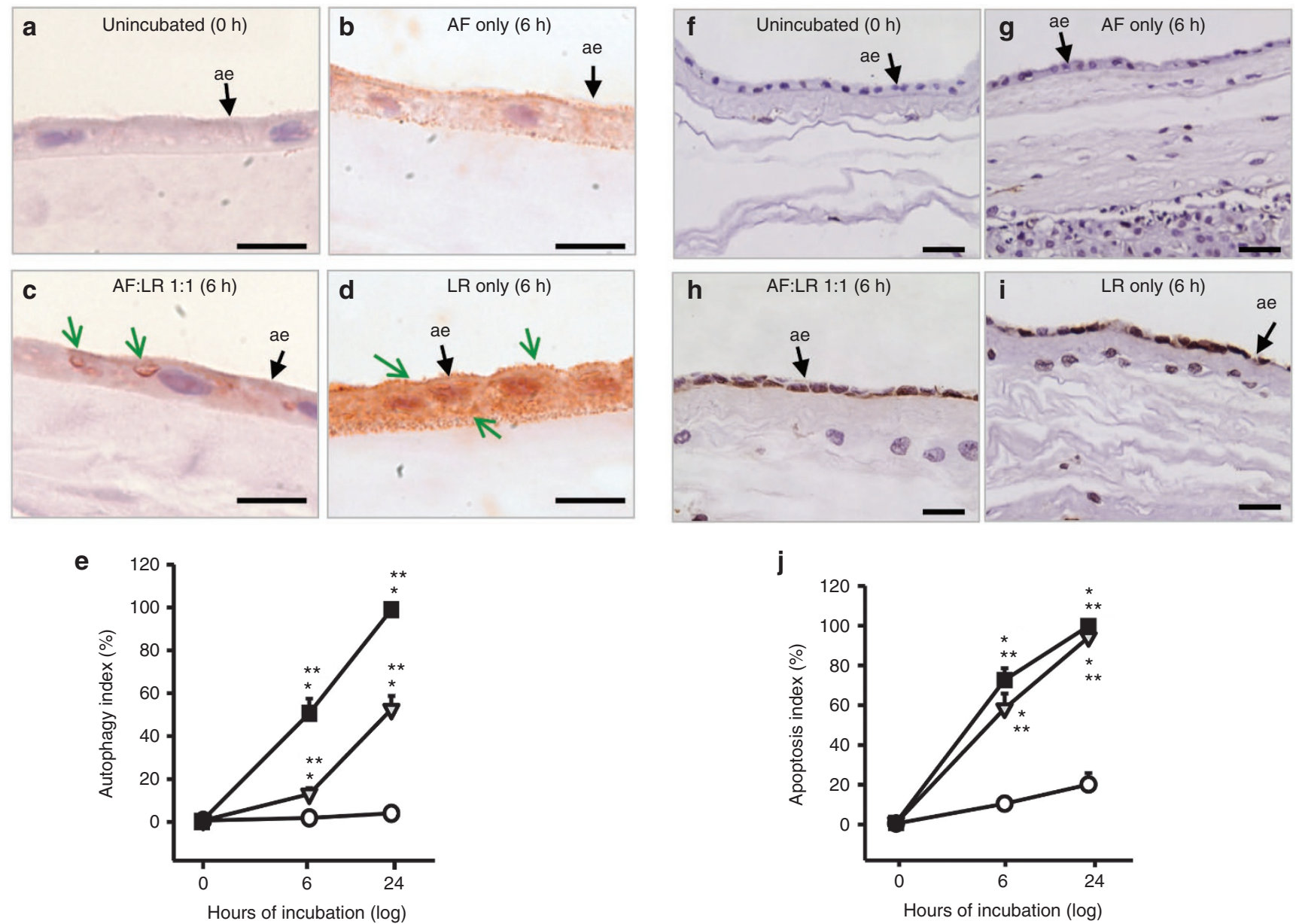

Figure 5. In-vitro study of autophagy and apoptosis in fetal membrane exposed to progressively diluted AF over 6 and 24 h. (a-e) Represents autophagy; (f-j) represents apoptosis; (a): Representative immunohistochemistry of amnion epithelial cells (ae) for baseline the autophagy marker LC3-II in an explant unincubated; (b) Represents for $6 \mathrm{~h}$ in native amniotic fluid with diffuse staining which represents LC3-I form; (c) Amnion epithelial cells of the fetal membranes incubated with AF diluted 1:1 with LR at $6 \mathrm{~h}$ showing autophagic puncta around the autophagosome (green arrow); (d) amniotic epithelial cells in fetal membranes of incubated in LR only at $6 \mathrm{~h}$ showing cells inundated with puncta and autophagosomes (green arrow); (e) Quantitative analysis of cells containing autophagic vacuoles (autophagy index) counted as percent of amniotic epithelial cells per field at 0,6 , and $24 \mathrm{~h}$ in explants $(n=6)$ cultured three different culture mediums; (f) TUNEL staining of amnion epithelial cells (ae) indicating levels of apoptosis in an unincubated explant. (g) Represents for $6 \mathrm{~h}$ in native amniotic fluid; (h) Amnion epithelial cells of the fetal membranes incubated with AF diluted 1:1 with LR at $6 \mathrm{~h}$ showing apoptotic cells (green arrow); (i) amniotic epithelial cells in fetal membranes of incubated in LR only at $6 \mathrm{~h}$ showing increased apoptotic cells (green arrow); (j) Apoptotic index at 0,6 , and $24 \mathrm{~h}$ in explants $(n=6)$ cultured three different culture mediums. Groups sharing at least one common red letter are not statistically different at $P>0.05$. (Data presented as mean $\pm \mathrm{SD}$ and analyzed by two-way repeated measured ANOVA. ${ }^{*} P<0.001$ vs. before incubation $(-\mathrm{O}-\mathrm{h}) ;{ }^{* * P}<0.001$ vs. incubation in native AF. Scale bar a-d: $25 \mu \mathrm{m}$ and $\mathbf{f}-\mathbf{i}: 50 \mu \mathrm{m}$. - Amniotic fluid only (AF Only); $\nabla-$ - Amniotic fluid: Lactated Ringer's solution in 1:1 concentration (AF:LR 1:1); - - Lacated Ringer's solution only (LR Only).

site of trocar insertion. When observed at delivery, the size of the defect was larger than the trocar size in over $50 \%$ of cases. The absence of the myofibroblasts at the site of injury suggests that the contraction phase of wound healing is not part of the reparative process of the injured fetal membranes; (ii) There was an increased cell death in the fetal membranes of the recipient with comparable apoptotic indices adjacent and at distance of the trocar penetration site. Yet, the rate of cell death in the donor membranes was comparable to control tissues, and significantly lower compared to all sites on the recipient's amniochorion; (iii) The collagen architecture of the recipient's fetal membranes was severely altered compared to the donor and reference membranes. This observation suggests that changes associated with either TTTS pathophysiology and/or iatrogenically-induced during the FLS procedure selectively impact on the viability and homeostasis of the recipient's fetal membranes. This premise argues that the local intra-amniotic milieu plays a much more significant role in the biology of fetal membranes compared to systemic or endocrine factors; (iv) The composition of the $\mathrm{AF}$ in the recipient sac was dramatically altered compared to normal pregnancy. This dilutional effect, likely secondary to TTTS pathophysiology, may cause shortage of essential nutrients to the fetal membranes which depend on the AF for survival (21). As demonstrated by our ex-vivo experiments, dilution of the AF alone was able to induce autophagy and cell death in cells of the fetal membranes that mimicked the level seen in vivo post-FLS. 
Our observation of a persistent trocar defect at delivery is consistent with the previous observation made by Gratacos et al. (12) who has examined the trocar defect site in 19 patients and the lack of cellular proliferation at the margin of the defect. Our study provides additional information by exploring for the first time additional histologic markers as well as sites remote from the site of defect. Moreover, the histologic changes on the remote sites point to a different process that appears to actively cause cell and tissue damage even to nonsurgically injured sites. A relevant question is why fetal membranes do not heal? One possible explanation is the avascular nature of the human fetal membranes. However, this is in contrast with the ability of other avascular biological tissues (i.e., cornea, tympanic membranes) to heal after an injury (16-18). In these tissues, wound repair involves cell proliferation and migration at the site of injury a phenomenon that involves adhesive molecules such as fibronectin, laminin, and complex signaling pathways such as epithelial growth receptor, fibroblast growth receptor, and JNK dependent pathway $(18,23)$. Why these mechanisms are downregulated or turned off in fetal membranes remains to be determined. Interestingly, amniotic membrane has been used to promote healing in clinical situations such as corneal burning and ulcerations, dural defects, intra-abdominal adhesions, peritoneal and genital reconstruction, hip arthroplasty, tendon and nerve repair, microvascular, oral and nasal reconstructive surgical procedures $(24,25)$. Both fresh and processed amniotic membranes appear to hold a variety of regulatory mediators that promote cellular proliferation, differentiation, and epithelialization $(26,27)$. From this perspective why fetal membranes are able to provide the necessary support to heterologous tissues to recover from damage but cannot repair themselves when injured remains a mystery that deserves further investigation.

In our study, the main cellular response of the recipient's sac fetal membranes, including the remote site from the trocar, was cell death through apoptosis. One possibility is that mechanical injury to the fetal membranes triggers a cascade of events yet to be determined that ultimately induces generalized cell death of the recipient's amniochorion; Alternatively or concurrent changes in the AF milieu due to the pathophysiology of the TTTS or related to amnioinfusion of LR solution during surgery may affect the concentration of essential nutrients required to sustain viability of the fetal membranes and in particular amnion $(10,21)$. The latter possibility is supported by our observation that the recipient's AF had significantly lower levels of glucose, proteins, and LDH levels, suggestive of polyhydramnios-induced dilution. It is thus plausible to propose that amnioinfusion with crystalloids such as LR or saline deprives the amnion from the nutritional support normally provided by AF initiating hence autophagy and ultimately cell death $(21,28)$.

Although fetal surgery has evolved remarkably as a field in the past 20 years, an effort to optimize the amnioinfusion fluid in an effort to sustain fetal membrane viability postsurgery had not been undertaken. Ringer's solution was originally developed in 1880 with the purpose of sustaining contractility of the frog's heart ex-vivo and was later modified by addition of lactate with the intention of treating acidosis (29). LR is now widely used as isotonic solution for intravenous administration. Yet the amnion is remarkably different than the blood vessel endothelium and may have different nutritional requirements. Interestingly, even when used for shock resuscitation, compared to resuscitation with whole blood, resuscitation with LR increased cellular apoptosis through a mechanism which involved upregulation of Bax protein (30).

The amnion layer of fetal membranes obtains its strength from that of the long mature collagen fibers in its compact layer (15). Disruption of the orientation and thickness of the collagen fibers noted in the recipient's sac could be explained by stretching beyond physiological limits due to polyhydramnios or defective collagen synthesis, turnover and degradation secondary to cell death. As previous studies have shown that in pregnancies complicated by PPROM and prior to the onset of labor cells in the fetal membranes undergo programmed cell death which parallel changes in collagen $(31,32)$, these mechanisms may play a role in the increased rate of PPROM and preterm labor after FLS.

A better understanding of fetal membrane biology following surgical interventions in-utero would shed light on the pathophysiology of PPROM and preterm delivery after FLS and other invasive fetal procedures such as myeolomenigocele repair (33), tracheal balloon occlusion for congenital diaphragmatic hernia (34), and selective reduction for complicated monochorionic twin gestations (35). Such knowledge would be the first step in considering surgical and therapeutic interventions aimed to prevent preterm delivery.

Strength of our study is inclusion of a large number of cases. One of the limitations was not gestational age matched controls for fetal membrane apoptotic index comparison. It is unethical deliver a patient without an indication at $31 \mathrm{wk}$. Although, we did not have the ability to examine the fetal membranes of all cases that had TTTS surgery, the number of cases systematically evaluated using histologic methods is the largest so far. Our novel findings that the surgical sac is injured the most are provocative and should stimulate research aimed to discover key therapeutic targets aimed to prevent apoptosis and collagen damage. It may as well initiate mechanistic research aimed to modify the intra- and postoperative conditions of fetal surgical interventions to prevent iatrogenic preterm birth and PPROM, which would improve significantly short and longterm neonatal outcomes.

\section{METHODS}

\section{Study Design and Patient Population}

The study protocols were approved by institutional review boards of University of Texas Medical School in Houston and of Yale University School of Medicine. All patients $(n=71)$ who were referred to the Fetal Center of University of Texas Medical School in Houston, TX from October 2011 to December 2012 with a monochorionic diamniotic gestation underwent a comprehensive ultrasound examination including Doppler studies for diagnosis and staging of TTTS based on the Quintero system (36). An informed consent was obtained prior to enrolling in the study. 
Fetoscopic laser ablation of the communicating vessels was performed through a single-port fetoscope under local anesthesia in conjunction with intravenous sedation. Ultrasound guided percutaneous entry was always into the recipient's sac using Seldinger's technique or direct trocar entry using 10 or 12 French Teflon cannula (Cook Medical, Bloomington, IN) as described in our previous publication (8). A diode laser (Dornier MedTech, Wessling, Germany) was used to ablate all vascular anastomoses using a selective sequential method. Amnioinfusion with LR was performed to improve visualization during fetoscopy. Chorio-amnion separation was assessed by ultrasound the following day after surgery.

Cases where fetal membranes appeared to have damaged significantly during delivery $(n=40)$ were excluded from the study. In the remaining 31 cases, the placenta and fetal membranes were examined after delivery to identify the recipient sac, donor sac, and intervening membranes. The recipient side of the sac was determined based on the side of the placenta where laser photocoagulation was performed, the larger size of the sac and of the placental surface (37). The fetoscopic entry site was identified by careful macroscopic survey of the fetal membranes.

Once identified, the trocar insertion site was measured in two perpendicular diameters and the area of the defect calculated from the ellipse formula. The defect was then excised along with $2 \mathrm{~cm}$ margins of full thickness fetal membrane. In a subset of 15 cases, additional biopsies of the fetal membranes $(4 \times 4 \mathrm{~cm})$ were obtained from a remote site in the recipient $\mathrm{sac}$ (at least $10 \mathrm{~cm}$ away from the site of defect), donor sac and inter-twin membrane.

For the histological analysis of our study, we used reference fetal membranes retrieved from a select group of 13 consecutive deliveries occurring in the same study period using the following inclusion criteria: singleton gestation, absent labor, absent histological chorioamnionitis and absent preeclampsia or maternal chronic medical conditions (i.e., diabetes, chronic hypertension, or collagen diseases). The GA at delivery for the singleton reference group was $34 \pm 2 \mathrm{wk}$, which was higher than post-FLS procedure in TTTS group $(31 \pm 5 \mathrm{wk}$; $P=0.01)$. GA and indications for delivery of reference group of patients were as follows: uterine scar dehiscence at $29 \mathrm{wk}(n=1)$, vasa previa at $32 \mathrm{wk}(n=4)$, placenta previa at $34 \mathrm{wk}(n=4)$, scheduled Cesarean section at $36-39 \mathrm{wk}(n=4)$ due to prior classical incision delivered $1 \mathrm{~d}$ after an amniocentesis for fetal lung maturity. For the segment of the study comparing AF composition, AF was collected from the recipient sac, prior to FLS procedure in 24 of the 71 TTTS cases treated in the study period. The AF was retrieved after trocar insertion but before any amnioinfusion or laser procedure was initiated. For reference group, we used AF retrieved from healthy pregnant women $(n=18)$ with singleton gestation who had a genetic amniocentesis to rule out aneuploidy. The GA at genetic amniocentesis group for this group was $20 \pm 2 \mathrm{wk}$ vs. GA pre-FLS procedure in TTTS group (21 $\pm 3 \mathrm{wk}, P=0.12)$.

\section{Histology and Immunohistochemistry}

Fetal membrane biopsies were placed in formalin and processed through paraffin embedding after careful orientation to allow for sectioning across all layers of the amniochorion. Paraffin tissue sections $(4 \mu \mathrm{m})$ were deparaffinized in xylene and rehydrated with graded ethanol to potassium-phosphate buffered saline solution (KPBS), pH 7.2. Slides were stained with hematoxylin \& eosin (H\&E) and Masson's trichrome (Richard-Allan Scientific, Kalamazoo, MI) to examine general tissue morphology. Circular polarized light microscopy after picrosirius red staining was performed to assess for collagen birefringence which represents an index of the organization and orientation of collagen fibers $(38,39)$ Digital images were acquired by using an Olympus U-STP polarizing light microscope equipped with an Olympus OLY-200 digital camera (Olympus, Melville, NY).

In preparation for immunohistochemistry, after deparaffinization and rehydration, heat-mediated antigen retrieval was performed with citrate buffer ( $\mathrm{pH}$ 6.0). Sections were peroxidase blocked and then incubated for $1 \mathrm{~h}$ with $2 \%$ normal donkey serum to block nonspecific binding. The following markers were sought: Ki-67 (marker of cellular proliferation), cytokeratin-7 (epithelial marker expressed by trophoblast cells), and vimentin (mesenchymal marker expressed by decidual cells). The following primary antibodies were used: anti-Ki67 polyclonal antibodies (1:200; Abcam, Cambridge, MA), monoclonal anticytokeratin-7 and anti-vimentin (1:100; Zymed/Invitrogen, Carlsbad, CA). Incubation with primary antibodies was done overnight at $4{ }^{\circ} \mathrm{C}$. Detection was performed with biotinylated donkey anti-mouse or anti-rabbit IgG (1:600; Jackson ImmunoResearch Laboratories, West Grove, PA) followed by avidin-biotin staining (Vectastain Elite ABC; Vector Laboratories, Burlingame, CA), Vector NovaRed (Vector Laboratories) as chromogen, and hematoxylin as counterstain. Slides where the first antibody was omitted or replaced by non-immune IgG served as negative controls.

Apoptosis (type I cell death) was assessed using ApopTag Peroxidase In Situ Apoptosis Detection Kit (EMO Millipore, Bellerica, MA). Representative areas of five high-power fields were examined in a blinded fashion. Cells displaying apoptotic features were differentiated from nonapoptotic cells. After counting the total numbers of cells per field an apoptotic index was calculated as the proportion of apoptotic cells to total number of cells counted. The apoptotic index was computed separately for amnion epithelium, amniotic mesenchymal cells and cEVTs. Autophagy is an adaptive catabolic process which allows survival during restricted nutrient conditions (40). Autophagy activity can be detected by immunohistochemistry of autophagosome-associated proteins such as microtubule-associated protein 1 light chain 3 (LC3). Changes in intracellular localization of LC3 staining from the diffuse intracytoplasmic form (LC3-I) to the autophagosome-associated form linked to phosphatidylethanolamine (LC3-II) appeared as localized distinct puncta around the nucleus $(41,42)$. A specific anti-LC3-II antibody (1:200; Abcam) was used to detect autophagic vacuoles. The autophagic index was calculated from the number of cells with LC3-II puncta relative to the number of amnion epithelial cells per high power field.

\section{Biochemical Analyses of AF}

Total protein was measured using the bicinchonic acid protein assay (Pierce Biotechnology, Rockford, IL). AF glucose concentration was measured using a quantitative enzyme-colorimetric assay (Stanbio, Boerne, TX). LDH was measured using a kinetic assay based on oxidation of lactate to pyruvate (LDH LiquiUV Assay, Stanbio).

\section{Fetal Membrane Explant System}

To determine the ability of AF dilution to induce cellular stress in the amniochorion, we collected full thickness fetal membranes from women pregnant with singletons who had a scheduled Cesarean delivery in the absence of labor ( $n=6$, GA $39 \pm 1 \mathrm{wk})$. Tissues were placed in organ culture exposed to their respective AF, LR or AF diluted 1:1 (v:v) with LR. Following incubation for 6 or $24 \mathrm{~h}$ tissues were harvested, formalin fixed, and paraffin embedded. Cell autophagy and apoptosis was evaluated by immunohistochemistry, as described above.

\section{Statistical Analysis}

The results were reported as either mean \pm SD or median with range, as appropriate. Data were compared with unpaired $t$-test, Mann-Whitney test, one-way or two-way ANOVA repeated measures ANOVA followed by post-hoc comparisons using Holm-Sidak method. A $P$ value of $<0.05$ was considered as significant. We used Statistical analyses were performed using STATA 12.1 (StataCorp, College Station, TX) and SigmaPlot version 12.0 statistical software (Systat, San Jose, CA).

\section{ACKNOWLEDGMENTS}

We acknowledge the support of referring physicians, nurses and coordinators in contributing towards the collection of specimens and clinical data.

\section{AUTHOR CONTRIBUTIONS}

R.P., L.K.M., and I.A.B. conceived, carried out experiments, and analyzed the data; K.J.M., T.K., A.J., and C.S.B. conceived experiments and analyzed data. E.G. carried out the experiments. All authors were involved in writing the paper and had final approval of the submitted and published versions.

\section{STATEMENT OF FINANCIAL SUPPORT}

The financial support for the research project was provided by the Department of Obstetrics, Gynecology and Reproductive Sciences at Yale 
University, New Haven, CT, and Department of Obstetrics, Gynecology and Reproductive Sciences at The University of Texas Medical School at Houston, TX.

Disclosures: None of the authors have any financial conflict of interest.

\section{REFERENCES}

1. Senat MV, Deprest J, Boulvain M, Paupe A, Winer N, Ville Y. Endoscopic laser surgery versus serial amnioreduction for severe twin-to-twin transfusion syndrome. N Engl J Med 2004;351:136-44.

2. Robyr R, Boulvain M, Lewi L, et al. Cervical length as a prognostic factor for preterm delivery in twin-to-twin transfusion syndrome treated by fetoscopic laser coagulation of chorionic plate anastomoses. Ultrasound Obstet Gynecol 2005;25:37-41.

3. Salomon LJ, Nasr B, Nizard J, et al. Emergency cerclage in cases of twin-totwin transfusion syndrome with a short cervix at the time of surgery and relationship to perinatal outcome. Prenat Diagn 2008;28:1256-61.

4. Lopriore E, Middeldorp JM, Sueters M, Oepkes D, Vandenbussche FP, Walther FJ. Long-term neurodevelopmental outcome in twin-to-twin transfusion syndrome treated with fetoscopic laser surgery. Am J Obstet Gynecol 2007;196:231.e1-4.

5. Habli M, Bombrys A, Lewis D, et al. Incidence of complications in twintwin transfusion syndrome after selective fetoscopic laser photocoagulation: a single-center experience. Am J Obstet Gynecol 2009;201:417.e1-7.

6. Rossi AC, Vanderbilt D, Chmait RH. Neurodevelopmental outcomes after laser therapy for twin-twin transfusion syndrome: a systematic review and meta-analysis. Obstet Gynecol 2011;118:1145-50.

7. Chavira ER, Khan A, Korst LM, Miller D, Goodwin TM, Chmait RH. Are patients with twin-twin transfusion syndrome and a very short cervix candidates for laser surgery? J Ultrasound Med 2009;28:633-9.

8. Papanna R, Mann LK, Johnson A, Sangi-Haghpeykar H, Moise KJ Jr. Chorioamnion separation as a risk for preterm premature rupture of membranes after laser therapy for twin-twin transfusion syndrome. Obstet Gynecol 2010;115:771-6.

9. Papanna R,MolinaS, Moise KY,Moise KJJr,Johnson A.Chorioamnion plugging and the risk of preterm premature rupture of membranes after laser surgery in twin-twin transfusion syndrome. Ultrasound Obstet Gynecol 2010;35:337-43.

10. Papanna R, Block-Abraham D, Mann LK, et al. Risk factors associated with preterm delivery after fetoscopic laser ablation for twin-twin transfusion syndrome. Ultrasound Obstet Gynecol 2014;43:48-53.

11. Chalouhi GE, Essaoui M, Stirnemann J, et al. Laser therapy for twin-totwin transfusion syndrome (TTTS). Prenat Diagn 2011;31:637-46.

12. Bourne GL. The microscopic anatomy of the human amnion and chorion. Am J Obstet Gynecol 1960;79:1070-3.

13. Niknejad H, Peirovi H, Jorjani M, Ahmadiani A, Ghanavi J, Seifalian AM. Properties of the amniotic membrane for potential use in tissue engineering. Eur Cell Mater 2008;15:88-99.

14. Orji FT, Agu CC. Determinants of spontaneous healing in traumatic perforations of the tympanic membrane. Clin Otolaryngol 2008;33:420-6.

15. Lou ZC. Spontaneous healing of traumatic eardrum perforation: outward epithelial cell migration and clinical outcome. Otolaryngol Head Neck Surg 2012;147:1114-9.

16. Chi C, Trinkaus-Randall V. New insights in wound response and repair of epithelium. J Cell Physiol 2013;228:925-9.

17. Danforth D, Hull RW. The microscopic anatomy of the fetal membranes with particular reference to the detailed structure of the amnion. Am J Obstet Gynecol 1958;75:536-47; discussion 548-50.

18. Bourne GL, Lacy D. Ultra-structure of human amnion and its possible relation to the circulation of amniotic fluid. Nature 1960;186:952-4.

19. Shen ZY, Xu LY, Li EM, et al. Autophagy and endocytosis in the amnion. J Struct Biol 2008;162:197-204.
20. White E, Lowe SW. Eating to exit: autophagy-enabled senescence revealed. Genes Dev 2009;23:784-7.

21. Thorburn A. Apoptosis and autophagy: regulatory connections between two supposedly different processes. Apoptosis 2008;13:1-9.

22. Vermeulen K, Van Bockstaele DR, Berneman ZN. Apoptosis: mechanisms and relevance in cancer. Ann Hematol 2005;84:627-39.

23. Rämet M, Lanot R, Zachary D, Manfruelli P. JNK signaling pathway is required for efficient wound healing in Drosophila. Dev Biol 2002;241: $145-56$.

24. Liu J, Sheha H, Fu Y, Liang L, Tseng SC. Update on amniotic membrane transplantation. Expert Rev Ophthalmol 2010;5:645-61.

25. Fairbairn NG, Randolph MA, Redmond RW. The clinical applications of human amnion in plastic surgery. J Plast Reconstr Aesthet Surg 2014;67:662-75.

26. Faulk WP, Matthews R, Stevens PJ, Bennett JP, Burgos H, Hsi BL. Human amnion as an adjunct in wound healing. Lancet 1980;1:1156-8.

27. Li W, He H, Chen YT, Hayashida Y, Tseng SC. Reversal of myofibroblasts by amniotic membrane stromal extract. J Cell Physiol 2008;215:657-64.

28. Shen ZY, Li EM, Lu SQ, et al. Autophagic and apoptotic cell death in amniotic epithelial cells. Placenta 2008;29:956-61.

29. Miller DJ. Sydney Ringer; physiological saline, calcium and the contraction of the heart. J Physiol 2004;555(Pt 3):585-7.

30. Deb S, Sun L, Martin B, et al. Lactated ringer's solution and hetastarch but not plasma resuscitation after rat hemorrhagic shock is associated with immediate lung apoptosis by the up-regulation of the Bax protein. J Trauma 2000;49:47-53; discussion 53-5.

31. Lei H, Furth EE, Kalluri R, et al. A program of cell death and extracellular matrix degradation is activated in the amnion before the onset of labor. J Clin Invest 1996;98:1971-8.

32. George RB, Kalich J, Yonish B, Murtha AP. Apoptosis in the chorion of fetal membranes in preterm premature rupture of membranes. Am J Perinatol 2008;25:29-32.

33. Adzick NS, Thom EA, Spong CY, et al.; MOMS Investigators. A randomized trial of prenatal versus postnatal repair of myelomeningocele. $\mathrm{N}$ Engl J Med 2011;364:993-1004.

34. Deprest J, Jani J, Gratacos E, et al.; FETO Task Group. Fetal intervention for congenital diaphragmatic hernia: the European experience. Semin Perinatol 2005;29:94-103.

35. Roman A, Papanna R, Johnson A, et al. Selective reduction in complicated monochorionic pregnancies: radiofrequency ablation vs. bipolar cord coagulation. Ultrasound Obstet Gynecol 2010;36:37-41.

36. Quintero RA, Morales WJ, Allen MH, et al. Staging of twin-twin transfusion syndrome. J Perinatol 1999;19:550-5.

37. Slaghekke F, Lewi L, Middeldorp JM, et al. Residual anastomoses in twintwin transfusion syndrome after laser: the Solomon randomized trial. Am J Obstet Gynecol 2014;211:285 e1-7.

38. Junqueira LC, Bignolas G, Brentani RR. Picrosirius staining plus polarization microscopy, a specific method for collagen detection in tissue sections. Histochem J 1979;11:447-55.

39. Buhimschi CS, Buhimschi IA, Yu C, et al. The effect of dystocia and previous cesarean uterine scar on the tensile properties of the lower uterine segment. Am J Obstet Gynecol 2006;194:873-83.

40. Mariño G, Niso-Santano M, Baehrecke EH, et al. Self-consumption: the interplay of autophagy and apoptosis. Nat Rev Mol Cell Biol 2014;15: $81-94$.

41. Rosenfeldt MT, Nixon C, Liu E, et al. Analysis of macroautophagy by immuno-histochemistry. Autophagy 2012;8:963-9.

42. Holt SV, Wyspianska B, Randall KJ, et al. The development of an immunohistochemical method to detect the autophagy-associated protein LC3-II in human tumor xenografts. Toxicol Pathol 2011;39:516-23. 